Original article

\title{
The influence of smoking on the pattern of disability and relapse risk in AQP4-positive Neuromyelitis Optica Spectrum Disorder, MOG-Ab Disease and Multiple Sclerosis
}

\author{
Silvia Messina ${ }^{\text {a,b,1 }}$, Romina Mariano ${ }^{\text {a, }}$, Ruth Geraldes ${ }^{\text {a,b, }}$, Su-Hyun Kim ${ }^{c}$, \\ Chanjira Satukijcha $^{\text {d,e }}$, Domizia Vecchio ${ }^{g}$, Yi Yi Chua ${ }^{f, g}$, James Taylor ${ }^{\text {f,g }}$, Naveen George ${ }^{f, g}$, \\ Ana Cavey $^{\mathrm{a}, \mathrm{b}}$, Alejandro Rubio Diaz ${ }^{\mathrm{a}}$, Sandra Reeve ${ }^{\mathrm{b}}$, Rosie Everett ${ }^{\mathrm{b}}$, Gabriele De Luca ${ }^{\mathrm{a}, \mathrm{b}}$, \\ Maria Isabel Leite ${ }^{\mathrm{a}, \mathrm{b}}$, Ho Jin Kim ${ }^{\mathrm{c}}$, Jacqueline Palace ${ }^{\mathrm{a}, \mathrm{b}}$," \\ ${ }^{\text {a }}$ Nuffield Department of Clinical Neurosciences, John Radcliffe Hospital, University of Oxford, Oxford, UK \\ ${ }^{\mathrm{b}}$ Department of Clinical Neurology, John Radcliffe Hospital, Oxford University Hospitals Trust, Oxford, UK \\ c Department of Neurology, Research Institute and Hospital of National Cancer, Goyang, Korea \\ ${ }^{d}$ Division of Neurology, Department of Medicine, Faculty of Medicine, Siriraj Hospital, Mahidol University, Bangkok, 10700, Thailand \\ ${ }^{\mathrm{e}}$ Bangkok Hospital Headquarters, BDMS, Bangkok, 10310, Thailand \\ ${ }^{\mathrm{f}}$ Department of Neurology, University of Piemonte Orientale, Novara, Italy \\ ${ }^{g}$ Oxford Medical School, University of Oxford, Oxford, UK
}

\section{A R T I C L E I N F O}

\section{Keywords:}

Neuromyelitis optica

MOGAD

Multiple sclerosis

Smoking

\begin{abstract}
A B S T R A C T
Background: the role of smoking on clinical outcomes of central nervous system (CNS) inflammatory disorders is unclear. To assess the effect of smoking on relapses and disability in neuromyelitis optica with aquaporin-4antibodies (NMOSD-AQP4-Ab), Myelin Oligodendrocyte Glycoprotein-antibodies associated disease (MOGAD) and relapsing remitting Multiple Sclerosis (MS) patients.

Methods: in a UK cohort of 101 NMOSD-AQP4-Ab, 70 MOGAD and 159 MS, and a Korean cohort of 97 NMOSD-AQ4-Ab, time to first relapse, annualised relapse rate, onset relapse severity and recovery, time to Expanded Disability Status Score(EDSS)/secondary progressive MS (SPMS) were compared between neversmokers and ever-smokers. All clinical data were collected under the local ethics between January 2017 and January 2019.

Results: Smoking did not affect the risk of relapse in any of the diseases. The risk of reaching EDSS 6.0 in the UK NMOSD-AQP4-Ab cohort was higher in ever smokers but this did not achieve significance (HR 2.12, $\mathrm{p}=0.068$ ). When combining the UK and Korea NMOSD-AQP4-Ab cohorts, poorer recovery from the onset attack was significantly more frequent in the ever-smokers versus the never smokers ( $55 \% \mathrm{vs} 38 \%, \mathrm{p}=0.04)$. In the MS cohort the risk of reaching EDSS 6 and SPMS was significantly higher in the ever-smokers $(\mathrm{HR}=2.67, \mathrm{p}=0.01$ and $\mathrm{HR}=3.18, \mathrm{p}=0.001$ ). In MOGAD similar patterns were seen without reaching significance.

Conclusions: In NMOSD-AQP4-Ab smoking associates with worse disability not through an increased risk of relapses but through poor relapse recovery. As in MS, smoking cessation should be encouraged in NMOSD-AQP4$\mathrm{Ab}$.
\end{abstract}

\section{Introduction}

Smoking associates with an earlier onset of neurological progression independent of relapses in Multiple Sclerosis (MS) (Wingerchuk, 2012; Healy et al., 2009). However, its effect on MS relapse rate is controversial (Petersen et al., 2019; Kvistad et al., 2016). It is not clear whether smoking affects inflammatory central nervous system (CNS) diseases more generally.

The neuromyelitis optica spectrum disorders (NMOSD) contains a mixed group of inflammatory CNS diseases with a predilection for the

\footnotetext{
* Corresponding authors.

E-mail address: jacqueline.palace@ndcn.ox.ac.uk (J. Palace).

1 These authors equally contributed to the manuscript.
} 
optic nerves and spinal cord: the majority has aquaporin-4 (AQP4) antibodies (Ab) targeting astrocytes which lead to demyelination as a secondary event (Lucchinetti et al., 2014). Some without AQP4-Ab have myelin-oligodendrocyte glycoprotein antibodies which cause a primary demyelinating disease (Spadaro et al., 2015) and the clinical phenotype can extend beyond NMOSD, thus this is now referred to as MOG antibody associated disease (MOGAD). The antibody negative NMOSD patients include a heterogeneous group of disorders; monophasic and chronic relapsing, some with MS, some with other inflammatory conditions and some which mimic AQP4-Ab disease (Kitley et al., 2013; Yeo et al., 2019). Thus, it is important to define patients according to their antibody specificity.

In contrast to MS, where disability predominantly occurs during the progressive phase and relapses are usually associated with mild disability, these antibody-mediated diseases have solely relapse-related accrual of disability. Additionally the nadir attack disability in both neuromyelitis optica associated with aquaporin-4 antibodies (NMOSDAQP4-Ab disease) and MOGAD is severe, and equally so, however MOGAD patients have better recovery (Kitley et al., 2014; Mariano et al., 2019). NMOSD-AQP4-Ab disease is a relapsing condition untreated, while MOGAD can be monophasic or relapsing with less long-term disability (Jurynczyk et al., 2017; Kitley et al., 2012).

There have been a few studies suggesting smoking may increase the risk of developing NMOSD (Eskandarieh et al., 2019, Simon et al., 2015, Y-jX et al., 2016, Laurent Kremer et al., 2015). Two abstracts reported analyses of the effect of smoking on disease severity in mixed NMOSD cohorts, one with a negative effect (Kremer L and Mealy, 2020) and one with an equivocal effect (Varela $F$ and Rojas, 2016). There are no papers on the influence of smoking on disease severity in NMOSD-AQP4-Ab and MOGAD defined cohorts.

We aim to determine the effect of smoking on time to clinical disability and how it relates to relapse frequency, severity and recovery, in three well-characterised disease cohorts: NMOSD-AQP4-Ab disease, MOGAD and relapsing-remitting MS.

\section{Methods}

\subsection{Study design, patients and clinical data}

This is a retrospective analysis of prospectively collected data exploring the effect of smoking on relapses and disability in patients with NMOSD-AQP4-Ab disease, MOGAD or relapsing remitting MS. Testing for the presence of AQP4-Ab and MOG-Ab was performed using current gold-standard cell-based assay techniques as described (Waters et al., 2012; Waters et al., 2015).

Data from three UK cohorts (NMOSD-AQP4-Ab disease, MOGAD and MS) were analysed. We included in the analysis NMOSD-AQP4, MS and MOGAD patients where details of their smoking history were recorded. Follow up data were collected up to January 2019.

A Korean cohort (AQP4-Ab positive) was used to validate the findings in NMOSD-AQP4-Ab disease in a different racial group.

All clinical data were collected according to local ethics, and included: smoking status (ever/never), age at disease onset, sex, disease duration (from onset to last follow up), disease course, treatment, time to first relapse, the number of relapses excluding the onset attack within the first 2 years from disease onset to give an annualised relapse rate, the time taken to reach the secondary progressive phase of MS ([SPMS], from onset to last follow up), the time taken to reach Expanded Disability Status Score (EDSS) 6.0 in NMOSD-AQP4-Ab disease and MS, and EDSS 4.0 in MOGAD, due to the rarity of higher levels of disability in the latter group. Time to analysis data are all from onset attack and assume patients were EDSS 0 just prior the onset attack.

Additionally, as relapses in the antibody-mediated diseases are severe and disability only related to such attacks, in the NMOSD-AQP4-Ab disease and MOGAD we collected data regarding the severity and recovery of the onset attack when it is assumed all patients start from a normal baseline state. Because the EDSS score is mainly affected by transverse myelitis (TM), the same data were analysed for the subgroup who had a TM at onset. The nadir severity of onset attacks were scored by the patients clinician as either mild (not affecting daily activities), or severe (affecting daily activities with or without dependence on others), and for recovery as good (good residual outcome or large improvement with independent function) or poor (no or mild improvement with poor residual outcome requiring some dependence on others).

Because relapse recovery is often poor in NMOSD-AQP4-Ab disease and thus we can more easily demonstrate the smoking related relapse effect on disability, we looked at a second group of NMOSD-AQP4-Ab disease from a different racial cohort. In MOGAD residual disability is low and in MS, where relapses are mild and not major contributors to disability, it is more difficult to detect the effect of individual relapses on disability.

Smoking data were collected either as part of the clinical history or using a questionnaire. Patients were classified as either ever-smokers (current smokers or ex-smokers of at least 10 cigarettes a day for at least 6 months) or never-smoker. Data on pack-year were not available.

\subsection{Statistical analysis}

Quantitative variables were described using mean, standard deviation and proportion. Differences between proportions (i.e. good vs poor recovery from onset) were calculated using chi-square.

We also produced a Kaplan-Meier survival curve to estimate time to the first relapse, time to reach EDSS 6.0 in the NMOSD-AQP4-Ab disease and MS groups, to reach EDSS 4.0 in the MOGAD group who rarely reach EDSS 6.0, and to reach SPMS in the MS group. A Cox proportional multivariate regression model was fitted using the outcomes above and including age, sex and smoking status as independent variables, to calculate the hazard ratio (HR). If there were significant treatment differences between ever smokers and never smokers, treatment was included in the model. In the absence of a discontinuation event, data were censored at the last observation day. The log-rank test of equality was used to compare categorical variables.

Data were analysed using STATA V.11.0 software packages (Stata Corp. 2011. Stata Statistical Software: Release 12. College Station TSL). $P$ values were 2 -tailed, and statistical significance was set at .05 .

\section{Results}

A total of 198 patients with NMOSD-AQP4-Ab disease (101 from the UK and 97 from Korea), 70 patients with MOGAD and 159 patients with MS were included. The demographics and smoking status of UK and Korea cohorts are shown in Table 1. Five patients in the Korean dataset did not have details of the severity and recovery of the onset attack.

\subsection{UK disease cohorts}

\section{The effect of smoking on NMOSD-AQP-Ab disease}

In the UK NMOSD-AQP4-Ab disease cohort, 31 were ever-smokers and 70 were never-smokers, with a higher proportion of Asian in the non-smokers group, but similar age, sex and disease duration. There was no difference in percentage of patients on immunosuppressive treatment (IST) (all were immunosuppressed) nor time to treatment.

The annualised relapse rate and time to first relapse, were not significantly different between ever-smokers and never-smokers (see Table 2).

The time to reaching EDSS 6.0, adjusting for age and sex, in the NMOSD-AQP4-Ab disease showed that the ever-smokers had a nonsignificant (trend level) double risk of reaching EDSS 6 when compared with the never-smokers (Cox proportional $\mathrm{HR}=2.12$, $\mathrm{p}=0.068,95 \%$ CI 0.99-4.75) as shown in the survival curve (see Fig. 1A).

Because disability was trending to be greater without affecting the risk of relapse, we explored the effect on severity and recovery from the 
Table 1

Demographic and clinical characteristics of the cohorts.

\begin{tabular}{|c|c|c|c|c|c|c|c|c|}
\hline & \multicolumn{4}{|c|}{ NMOSD-AQP4-Ab disease } & \multicolumn{2}{|l|}{ MS } & \multicolumn{2}{|l|}{ MOGAD } \\
\hline & \multicolumn{2}{|l|}{ UK cohort } & \multicolumn{2}{|l|}{ Korea cohort } & \multicolumn{2}{|l|}{ UK cohort } & \multicolumn{2}{|l|}{ UK cohort } \\
\hline & $\begin{array}{l}\text { ever-smokers } \\
(\mathrm{n}=31)\end{array}$ & $\begin{array}{l}\text { never-smokers } \\
(\mathrm{n}=70)\end{array}$ & $\begin{array}{l}\text { ever-smokers } \\
(\mathrm{n}=17)\end{array}$ & $\begin{array}{l}\text { never-smokers } \\
(\mathrm{n}=80)\end{array}$ & $\begin{array}{l}\text { ever-smokers } \\
(\mathrm{n}=53)\end{array}$ & $\begin{array}{l}\text { never- smokers } \\
(\mathrm{n}=106)\end{array}$ & $\begin{array}{l}\text { ever smokers } \\
(\mathrm{n}=27)\end{array}$ & $\begin{array}{l}\text { never-smokers } \\
(\mathrm{n}=43)\end{array}$ \\
\hline Female, n (\%) & $25(81)$ & $54(77)$ & $7(41)$ & $75(94)$ & $43(81)$ & $86(81)$ & $9(33.3)$ & $30(70)$ \\
\hline \multicolumn{9}{|l|}{ Ethnicity, \% } \\
\hline White & $16(52)$ & $39(56)$ & 0 & 0 & $49(92)$ & $98(92)$ & $21(78)$ & 34 (79) \\
\hline Asian & $1(3)$ & $9(13)$ & $17(100)$ & $80(100)$ & 0 & 0 & $2(7)$ & $5(12)$ \\
\hline Black-Caribbean & $7(22)$ & $11(16)$ & 0 & 0 & 0 & 0 & $1(4)$ & $1(2)$ \\
\hline Mixed & $3(10)$ & $1(1)$ & 0 & 0 & 0 & 0 & 0 & $2(5)$ \\
\hline Unknown & $4(13)$ & $10(14)$ & 0 & 0 & $4(8)$ & $8(8)$ & $3(11)$ & $1(2)$ \\
\hline Mean age at onset \pm SD & $47.8 \pm 13.5$ & $48.6 \pm 17.6$ & $35.2 \pm 12.7$ & $33.4 \pm 12.1$ & $32.6 \pm 7.8$ & $34.7 \pm 7.4$ & $34.7 \pm 13.5$ & $33.3 \pm 14.1$ \\
\hline $\begin{array}{l}\text { Mean disease Duration } \\
\pm \mathrm{SD} \text { (years) }\end{array}$ & $7.0 \pm 6.6$ & $7.8 \pm 7.5$ & $9.2 \pm 4.8$ & $10.8 \pm 5.1$ & $13.3 \pm 8$ & $12.4 \pm 8.9$ & $4.9 \pm 8.4$ & $4.4 \pm 5.7$ \\
\hline
\end{tabular}

Legend: SD = standard deviation.

onset attack. The frequency of severe onset attacks did not differ (84\% ever smokers vs $79 \%$ never smokers). However, in ever smokers versus never smokers a greater proportion of TM onset attacks were severe $(92 \%$ vs $68 \%)$ and a greater proportion of attacks had poor recovery ( $65 \%$ versus $50 \%$ of all attacks, $62 \%$ versus $44 \%$ of TM attacks), without reaching significance (see Fig. 2).

\subsection{The effect of smoking on MOGAD}

In the MOGAD cohort, 27 were ever-smokers and 43 never-smokers, with a higher proportion of females in the never-smokers group, and similar age and disease duration.

There was no difference in IST (percentage on treatment or time to treatment) in smokers or non-smokers.

The annualised relapse rate and the time to first relapse were not significantly different between the ever-smokers and never-smokers (see Table 2).

The time to reach EDSS 4, adjusting for age and sex, between the two groups is not significantly different (see Fig. 1B). Although the number of patients reaching this endpoint was very low overall, more smokers did reach EDSS 4 (see Table 2 and Fig. 1B).

The frequency of severe onset attack did not differ $(70 \%$ in both eversmokers and never-smokers). However, in ever-smokers versus neversmokers, a higher proportion of TM onset attack were severe $(70 \%$ vs $50 \%$ ) and a higher proportion had poor recovery (22\% vs $9 \%$ all onset attack; $30 \%$ vs $25 \%$ TM onset attack) without reaching significance (see Fig. 2).

\subsection{The effect of smoking on $M S$}

In the MS cohort, 53 were ever-smokers and 106 were never-smoker with similar age, sex proportion and disease duration.

There were more non-smokers treated at some point with DMTs (71 patients [67\%] vs 25 patients [47\%], $\mathrm{p}=0.02$ ), thus, we included the status ever treated with disease modifying therapy (DMT) as a covariate, along with age and sex.

The annualised relapse rate and the time to first relapse between the ever-smokers and never-smokers was not significantly different (see Table 2).

The risk of reaching EDSS 6.0 and the secondary progressive stage, adjusting for age, sex and treatment, was higher in the ever-smokers, (EDSS 6 Cox proportional $\mathrm{HR}=2.67, \mathrm{p}=0.01,95 \% \mathrm{CI} 1.3-5.6$ and secondary progressive MS Cox proportional $\mathrm{HR}=3.18, \mathrm{p}=0.001,95 \% \mathrm{CI}$ 1.6-6.4) (see Fig. 1C).

As attack severity is usually mild in MS, not yet diagnosed MS patients were usually assessed in out-patients by the neurologist months after the episode and thus we did not have detailed examination of onset attack severity.

\subsection{Further investigation into the effect of smoking on relapse severity and recovery: Korean and UK NMOSD-AQP4-Ab disease cohorts}

Due to the ability to differentiate relapse frequency, relapse severity and recovery and disability in NMOSD-AQP4-Ab patients (MS relapses mainly mild and MOGAD disability usually mild), we added in a new NMOSD-AQP4-Ab disease cohort from Korea to study this further.

\subsection{Korean NMOSD-AQP4-Ab disease cohort}

In the Korean cohort, 17 patients were ever-smokers and 80 patients were never-smokers with a higher proportion of female in the neversmokers, but similar age and disease durations. There was no difference in percentage of patients on IST (all were immunosuppressed) nor time to treatment.

The annualised relapse rate, and the time to relapse was not different between ever-smokers and never-smokers (see Table 2).

There was no difference in the time to reach EDSS 6.0, adjusting for age and sex, between the two groups (see Table 2).

There were no differences in the severity of the onset attack between ever-smokers and never-smokers, as all the patients in both groups experienced a severe onset attack. There was a higher proportion of patients with poor recovery from the onset attack in the ever-smokers versus never-smokers ( $38 \%$ vs $26 \%$ all onset attack; $38 \%$ vs $15 \% \mathrm{TM}$ onset attack) (see Fig. 2) although this did not reach significance.

\subsection{Combined UK-Korean NMOSD-AQP4-Ab disease cohort}

To increase the number, we combined the NMOSD-AQP4-Ab disease cohorts to see if trends became significant and to see if we could detect an effect of current versus ex-smokers.

Again, no effect on relapse frequency nor time to relapse was seen (see Table 2).

We found a significantly poorer recovery from onset attacks in eversmokers compared to the never-smoker group $(55 \%$ versus $38 \%, \mathrm{p}=$ 0.04) (Fig. 2) and a greater percentage of current smokers had a poor recovery from onset attacks than past-smokers than never smokers although at a trend level (65\% versus $50 \%$ versus $38 \% \mathrm{p}=0.06$ ) .

The time to reach EDSS 6 between current, ex-smokers and nonsmokers, also showed a non-significant but graded response with shorter times to EDSS 6.0 for current versus past versus never smokers (log-rank $\mathrm{p}=0.097$ ) (see Fig. 1D).

\section{Discussion}

This is the first paper of patients with NMOSD-AQP4-Ab defined disease and MOGAD that describes the effect of smoking on relapse and disability, side by side to relapsing remitting MS (see Table 3). It appears 


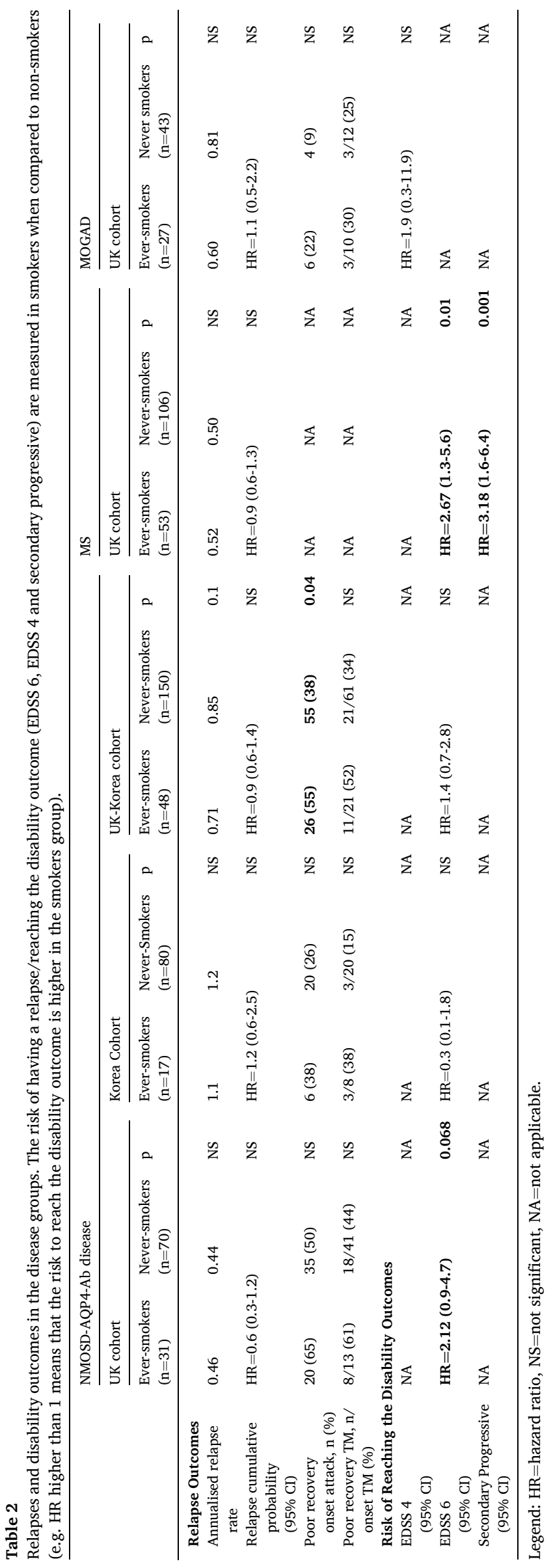

consistent across all diseases that smoking does not associate with an increased risk of relapse as defined by time to first relapse from onset and relapse rate. However, recovery from relapses in the antibody cohorts (using the onset attack as a surrogate) in ever smokers was less frequent. Smoking did appear to influence disability with ever-smokers having an increased risk of reaching EDSS 6 and the secondary progressive phase in the MS cohort. There is a trend for an increased risk to reach EDSS 6 in the NMOSD-AQP4-Ab cohort, although not significant and not confirmed in the Korean cohort. As expected, disability was low in the MOGAD group, and smoking had no significant effect although more ever-smokers did reach EDSS 4 than non-smokers. Thus, any increase in disability across the three inflammatory diseases seems to be related to a poorer recovery, and not to an increased number relapse.

Two previous abstracts reported the effect of smoking on outcomes in mixed NMOSD cohorts 17, 18: a multicentre study suggested that current smokers experience a higher number of relapses and more severe attacks when compared with non-smokers and a correlation of smoking with EDSS (Kremer L and Mealy, 2020). The second reported a positive correlation between smoking and EDSS at diagnosis and a negative correlation with visual acuity, and the effect on EDSS was removed with treatment (Varela F and Rojas, 2016). Their cohorts will have contained NMOSD-AQP4-Ab disease, MOGAD and antibody negative diseases and it is difficult to directly compare our defined cohort results with those reported in these abstracts. Furthermore, in our analysis we adjusted for age and sex.

In our UK NMOSD-AQP4-Ab disease cohort we found that smokers reached EDSS 6.0 more quickly although significance was not reached $(\mathrm{p}=0.068)$ with a hazard ratio of just over double, but without any increase in the number of relapses. In both NMOSD-AQP4-Ab disease cohorts, a higher percentage of never-smokers had a good recovery, and this reached significance when the UK and Korean cohorts were combined to increase the sample size. We did not find a significant effect of smoking on reaching EDSS 6 in the Korean cohort, however few patients in this cohort smoked nor reached EDSS 6 which itself might be explained by fewer smokers and a milder disease phenotype as has previously been shown in a comparative study of ethnicity (Kim et al., 2018). Overall, it appears that smoking in NMOSD-AQP4-Ab disease leads to worse disability not by increasing relapse numbers but by reducing relapse recovery, and this was further supported by the observation that poor relapse recovery was more frequent in current-smokers ( $16 \%$ of our UK NMOSD cohort [15 out of 90] almost in line with the UK national data) (AshitU 2019) than in past-smokers and never-smokers (at a trend level).

In the MOGAD cohort, relapse risk was also not influenced by smoking. Because MOGAD is a milder disease with better relapse recovery and less disability accumulation (Mariano et al., 2019), we did not observe a significant impact on these outcomes. However, the same pattern as in NMOSD-AQP4-Ab disease was seen with more patients in the ever-smoking group reaching EDSS 4 and having a poor recovery.

In the MS cohort, the relatively high relapse rate is likely due to the fact that this is calculated for the first two years of disease when most patients would not start treatment until the second attack. Again, in the MS group relapse risk was not affected by smoking, but it increased by three times the risk of conversion to the secondary progressive stage of the disease and by two times the risk of reaching EDSS 6 . These findings are in line with previous studies and confirm the detrimental effect of smoking on the accrual of disability (Degelman and Herman, 2017; Geraldes et al., 2017). In MS the disability from relapses is small and the majority of disability is accrued during the progressive phase thus, we hypothesise that in MS smoking detrimentally affects either recovery from or damage due to the pathological processes involved in progression.

Overall, our study shows a consistent pattern of worse outcomes in these inflammatory demyelinating conditions, which appears to be predominantly driven by greater tissue damage and/or poorer recovery, rather than by triggering relapses. Many mechanisms of the effect of 


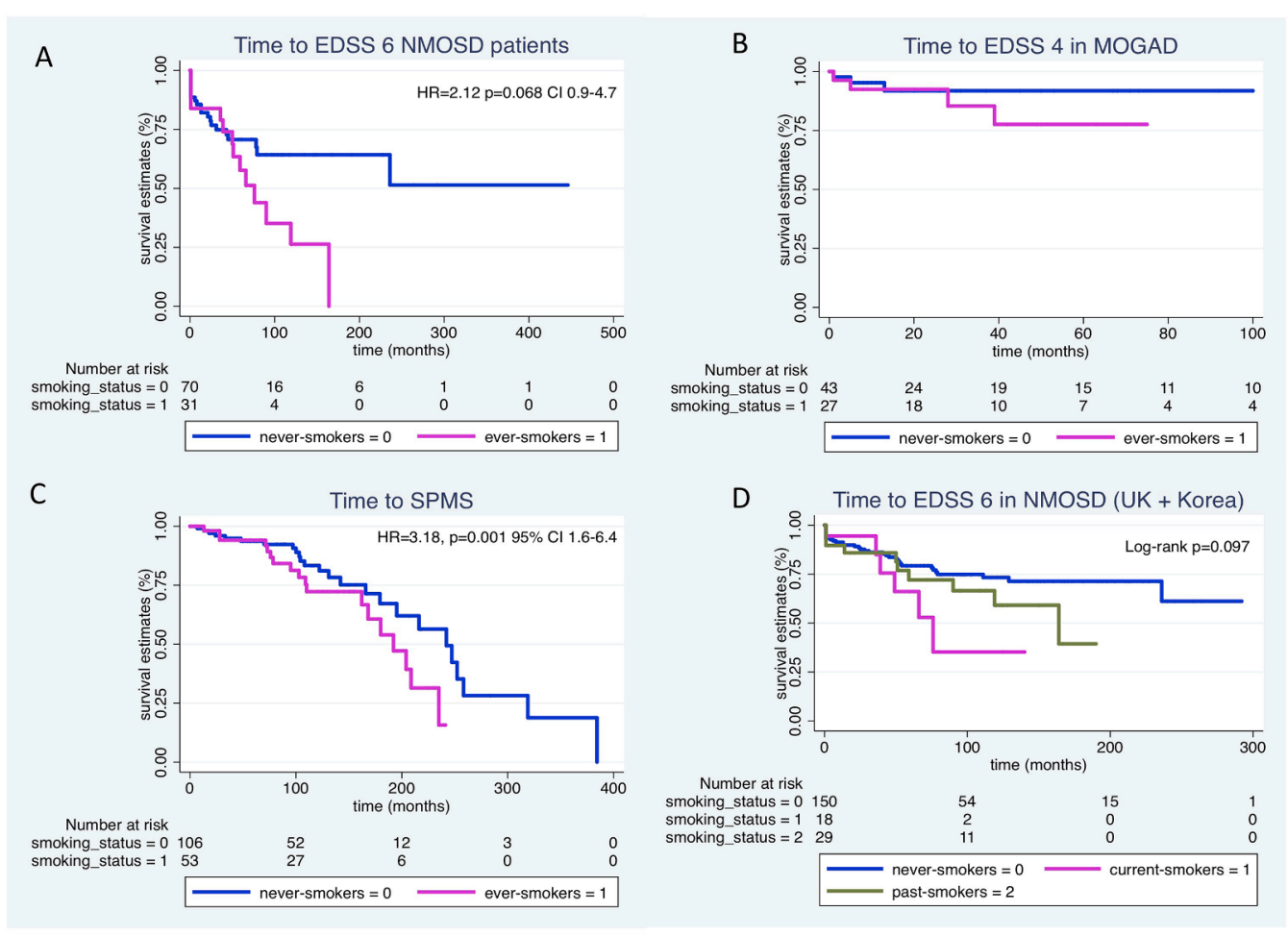

Fig. 1. Kaplan-Meier analysis of (A) Time to EDSS 6 in the UK NMOSD-AQP4-Ab disease cohort, (B) Time to EDSS 4 in the UK MOGAD cohort, (C) Time to SPMS in the UK MS cohort and Time to EDSS 6 in the combined UK and Korean NMOSD-AQP4-Ab disease cohort.

\section{Percentage of poor recovery from onset attack}

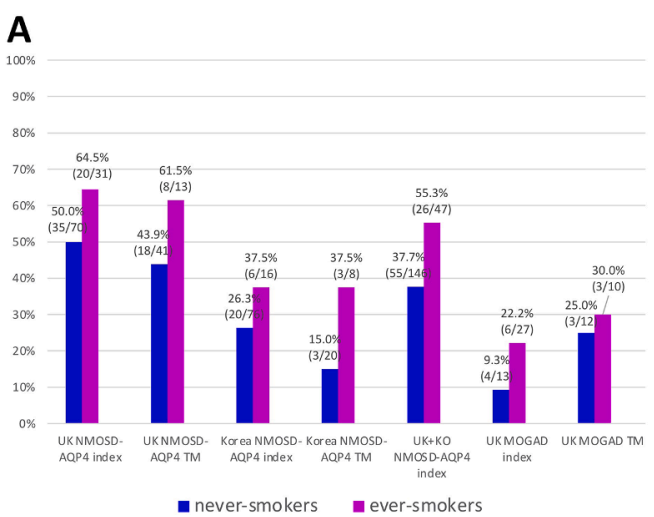

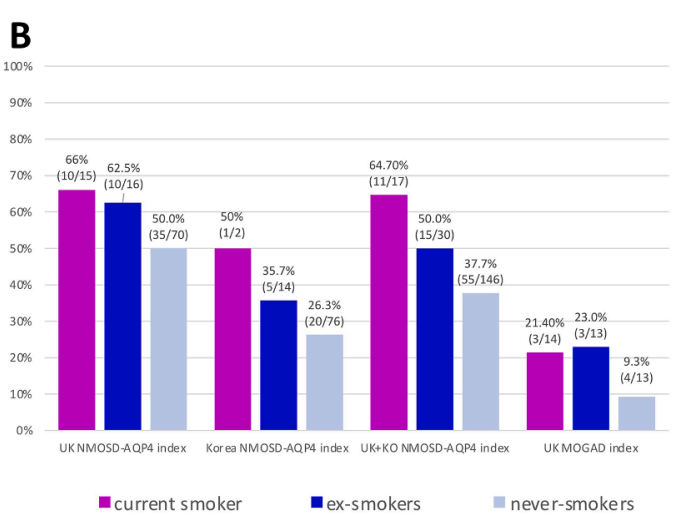

Fig. 2.A. Percentage comparison between never-smokers and ever-smokers of patients with poor recovery in the UK and Korean (KO) AQP4-Ab and the UK MOG-Ab cohorts from their onset attack or TM at onset. 2.B Percentage comparison between current-smokers, ex-smokers and never-smokers, of patients with poor recovery in the UK and Korea NMOSD-AQP4-Ab disease cohorts, the combined UK and Korean NMOSD-AQP4-Ab disease cohort and the UK MOGAD cohort from their onset attack.
Table 3

Summary table of the effects of smoking in the three diseases group on the three outcomes.

\begin{tabular}{llll}
\hline & Relapse Risk & Attack Recovery & Disability \\
\hline AQP4-Ab disease & $\leftrightarrow$ & ++ & + \\
MOG-Ab disease & $\leftrightarrow$ & + & + \\
MS & $\leftrightarrow$ & NM & ++ \\
\hline
\end{tabular}

$\overline{\text { Legend } \leftrightarrow=\text { No effect; }+=\text { Non-significant pattern; }++=\text { significant effect; }}$ $\mathrm{NM}=$ not measured.

smoking on pathology have been reported (Rosso and Chitnis, 2019). The increased activation in immune function reported with smoking (Alrouji et al., 2019) could be offset by its effect on reducing immune triggering events such as activation of macrophages, antigen presenting cells (APC), pro-B and pre-B cells with a decrease in the production of antibodies (Metcalfe et al., 2014; Brandsma et al., 2009). However, once an acute inflammatory event has occurred, smoking may lead to greater damage and poorer recover by increasing pro-inflammatory cytokines linked with neurodegeneration (Petrescu et al., 2010; Ribeiro et al., 2019), by gas related damage (such as cyanide, nitric oxide and carbon monoxide) which may lead to hypoxia, vascular injury (Spencer et al., 2018), mitochondrial damage and oligodendrocytes necrosis (Valavanidis et al., 2009; Mitrovic et al., 1995), impairing remyelination, increasing axonal loss and reducing recovery (Shirani and Tremlett, 2010; Yin et al., 2015). Additionally, cigarette smoke has been reported to increase complement activation (Yin et al., 2015; Yuan et al., 2015) which could also increase tissue damage once the inflammatory response has been activated.

This study is limited by the lack of quantification of the number of cigarettes per day, because this was a retrospective analysis of data already collected rather than a prospective study. Additionally, our definition of never-smokers allowed inclusion of those who had smoked very small amounts (less than 10 cigarettes per day for less than 6 
months) and this could have slightly worsened the outcome of the neversmoker group. However, when dividing ever smokers into current and ex-smokers there did appear to be a graded detrimental effect in both the NMOSD UK and Korea cohorts to support our observations. The pattern of the finding of no effect on relapses could be missed due to small sample sizes. However, the observation was consistently observed across all the subgroups and relapse outcomes are generally more sensitive than disability and progression outcomes in clinical trials. Thus, it is likely our observations are real. The results of this study with a similar pattern observed across the three diseases should lead to a future prospective study designed to collect a more detailed smoking and vascular history.

In conclusion, we demonstrate the negative impact of smoking on demyelinating disorders of the central nervous system. We therefore believe that smoking cessation should be actively encouraged in these disease groups and patients should be supported in achieving this as part of their clinical management.

\section{CRediT authorship contribution statement}

Silvia Messina: Formal analysis, Methodology, Writing - original draft. Romina Mariano: Formal analysis, Writing - original draft. Ruth Geraldes: Methodology, Writing - original draft. Su-Hyun Kim: Validation, Resources, Writing - review \& editing. Chanjira Satukijcha: Resources. Domizia Vecchio: Resources. Yi Yi Chua: Resources. James Taylor: Resources. Naveen George: Resources. Ana Cavey: Project administration. Alejandro Rubio Diaz: Resources, Data curation. Sandra Reeve: Data curation, Resources. Rosie Everett: Data curation, Resources. Gabriele De Luca: Resources, Supervision. Maria Isabel Leite: Supervision. Ho Jin Kim: Supervision. Jacqueline Palace: Conceptualization, Supervision, Writing - review \& editing.

\section{Declaration of Competing Interests}

Dr Messina reported receiving travel grants from Biogen, Novartis, Bayer, Merck \& Co, Roche and Almirall and honorarium from Biogen for advisory work. Dr Mariano reported receiving funding from the Rhodes Trust. Dr Geraldes has received support for scientific meetings and courses or honoraria for advisory work from Wolfson College, by the EAN, Bayer, Biogen, Merck and Novartis. Dr De Luca is supported by the NIHR Biomedical Research Centre (BRC), Oxford and has research funding from the Oxford BRC, MRC (UK) and Merck-Serono. He has received travel expenses from Bayer Schering, Biogen Idec, Genzyme, Merck Serono and Novartis and honoraria as an invited speaker for Bayer Schering and Novartis. Dr. Leite reports funding from NHS National Specialised Commissioning Group for Neuromyelitis optica, UK, and the NIHR Oxford Biomedical Research Centre, UK; and speaker honoraria or travel grants from Biogen Idec, Novartis, and the GuthyJackson Charitable Foundation. Dr Kim received a grant from the National Research Foundation of Korea; received consultancy/speaker fees from Alexion, Aprilbio, Celltrion, Eisai, HanAll BioPharma, Merck Serono, Novartis, Sanofi Genzyme, Teva-Handok, and Viela Bio; serves on a steering committee for MedImmune/Viela Bio; is a co-editor for the Multiple Sclerosis Journal and an associated editor for the Journal of Clinical Neurology. Dr Palace reported receiving grants from National Health Service to conduct a national congenital myasthenia service and neuromyelitis service, the Multiple Sclerosis Society, Guthie Jackson Foundation, Chugai, and MedImmune; personal fees from Teva, Novartis, Roche, MedDay, and ARGENX; and grants and personal fees from Merck Serono, Biogen, Alexion, and ABIDE, Genzyme, and MedImmun. No other disclosures were reported.

\section{Acknowledgements}

We gratefully acknowledge the Highly Specialised Commissioning Team of the National Health Service for funding the Neuromyelitis
Optica service in Oxford. The authors received no financial support for the research, authorship, and/or publication of this article.

\section{References}

Alrouji, M, Manouchehrinia, A, Gran, B, et al., 2019. Effects of cigarette smoke on immunity, neuroinflammation and multiple sclerosis. J. Neuroimmunol. 329, 24-34. https://doi.org/10.1016/j.jneuroim.2018.10.004, 2018/10/27.

2019 AshitU.

Brandsma, CA, Hylkema, MN, Geerlings, M, et al., 2009. Increased levels of (class switched) memory B cells in peripheral blood of current smokers. Respir. Res. 10 (108) https://doi.org/10.1186/1465-9921-10-108, 2009/11/17.

Degelman, ML, Herman, KM., 2017. Smoking and multiple sclerosis: A systematic review and meta-analysis using the Bradford Hill criteria for causation. Mult. Scler. Relat. Disord. 17, 207-216. https://doi.org/10.1016/j.msard.2017.07.020, 2017/10/23.

Eskandarieh, S, Moghadasi, AN, Sahraiain, MA, et al., 2019. Association of cigarette smoking with neuromyelitis optica-immunoglobulin G sero-positivity in neuromyelitis optica spectrum disorder. Iran. J. Neurol. 18, 93-98, 2019/11/22.

Geraldes, R, Esiri, MM, DeLuca, GC, et al., 2017. Age-related small vessel disease: a potential contributor to neurodegeneration in multiple sclerosis. Brain Pathol. 27, 707-722. https://doi.org/10.1111/bpa.12460, 2016/11/20.

Healy, BC, Ali, EN, Guttmann, CR, et al., 2009. Smoking and disease progression in multiple sclerosis. Arch. Neurol. 66, 858-864. https://doi.org/10.1001/ archneurol.2009.122, 2009/07/15.

Jurynczyk, M, Messina, S, Woodhall, MR, et al., 2017. Clinical presentation and prognosis in MOG-antibody disease: a UK study. Brain 140, 3128-3138. https://doi. org/10.1093/brain/awx276, 2017/11/15.

Kim, SH, Mealy, MA, Levy, M, et al., 2018. Racial differences in neuromyelitis optica spectrum disorder. Neurology 91, e2089-e2099. https://doi.org/10.1212/ WNL.0000000000006574, 2018/10/28.

Kitley, J, Leite, MI, Kuker, W, et al., 2013. Longitudinally extensive transverse myelitis with and without aquaporin 4 antibodies. JAMA Neurol. 70, 1375-1381. https:// doi.org/10.1001/jamaneurol.2013.3890, 2013/09/04.

Kitley, J, Leite, MI, Nakashima, I, et al., 2012. Prognostic factors and disease course in aquaporin-4 antibody-positive patients with neuromyelitis optica spectrum disorder from the United Kingdom and Japan. Brain 135, 1834-1849. https://doi.org/ 10.1093/brain/aws109, 2012/05/12.

Kitley, J, Waters, P, Woodhall, M, et al., 2014. Neuromyelitis optica spectrum disorders with aquaporin-4 and myelin-oligodendrocyte glycoprotein antibodies: a comparative study. JAMA Neurol. 71, 276-283. https://doi.org/10.1001/ jamaneurol.2013.5857, 2014/01/16.

Kremer L, AN, Mealy, M, et al., 2020. Tobacco smoking and severity of neuromyelitis optica. Multiple Sclerosis J. 21, 458.

Kvistad, S, Myhr, KM, Holmoy, T, et al., 2016. No association of tobacco use and disease activity in multiple sclerosis. Neurol. Neuroimmunol. Neuroinflamm. 3, e260. https://doi.org/10.1212/NXI.0000000000000260, 2016/07/28.

Laurent Kremer, NA, Mealy, Maureen, Mutch, Kerry, Lewy, Michael, Jacob, Anu, Collongues, Nicolas, De Seze, Jerome, 2015. Tobacco smoking and severity of neuromyelitis optica. Neurology 84, 14 Supplement.

Lucchinetti, CF, Guo, Y, Popescu, BF, et al., 2014. The pathology of an autoimmune astrocytopathy: lessons learned from neuromyelitis optica. Brain Pathol. 24, 83-97. https://doi.org/10.1111/bpa.12099, 2013/12/19.

Mariano, R, Messina, S, Kumar, K, et al., 2019. Comparison of clinical outcomes of transverse myelitis among adults with myelin oligodendrocyte glycoprotein antibody vs aquaporin-4 antibody disease. JAMA Netw. Open 2, e1912732. https:// doi.org/10.1001/jamanetworkopen.2019.12732, 2019/10/10.

Metcalfe, HJ, Lea, S, Hughes, D, et al., 2014. Effects of cigarette smoke on Toll-like receptor (TLR) activation of chronic obstructive pulmonary disease (COPD) macrophages. Clin. Exp. Immunol. 176, 461-472. https://doi.org/10.1111/ cei.12289, 2014/02/18.

Mitrovic, B, Ignarro, LJ, Vinters, HV, et al., 1995. Nitric oxide induces necrotic but not apoptotic cell death in oligodendrocytes. Neuroscience 65, 531-539. https://doi. org/10.1016/0306-4522(94)00491-m, 1995/03/01.

Petersen, ER, Sondergaard, HB, Laursen, JH, et al., 2019. Smoking is associated with increased disease activity during natalizumab treatment in multiple sclerosis. Mult. Scler. 25, 1298-1305. https://doi.org/10.1177/1352458518791753, 2018/08/03.

Petrescu, F, Voican, SC, Silosi, I., 2010. Tumor necrosis factor-alpha serum levels in healthy smokers and nonsmokers. Int. J. Chron. Obstruct. Pulmon. Dis. 5, 217-222. https://doi.org/10.2147/copd.s8330, 2010/08/18.

Ribeiro, CM, Oliveira, SR, Alfieri, DF, et al., 2019. Tumor necrosis factor alpha (TNFalpha) and its soluble receptors are associated with disability, disability progression and clinical forms of multiple sclerosis. Inflamm. Res. 68, 1049-1059. https://doi. org/10.1007/s00011-019-01286-0, 2019/09/29.

Rosso, M, Chitnis, T, 2019. Association Between Cigarette Smoking and Multiple Sclerosis: A Review. JAMA Neurol. https://doi.org/10.1001/jamaneurol.2019.4271, 2019/12/17.

Shirani, A, Tremlett, H., 2010. The effect of smoking on the symptoms and progression of multiple sclerosis: a review. J. Inflamm. Res. 3, 115-126. https://doi.org/10.2147/ JIR.S12059, 2010/01/01.

Simon, KC, Schmidt, H, Loud, S, et al., 2015. Risk factors for multiple sclerosis, neuromyelitis optica and transverse myelitis. Mult. Scler. 21, 703-709. https://doi. org/10.1177/1352458514551780, 2014/10/12.

Spadaro, M, Gerdes, LA, Mayer, MC, et al., 2015. Histopathology and clinical course of MOG-antibody-associated encephalomyelitis. Ann. Clin. Transl. Neurol. 2, 295-301. https://doi.org/10.1002/acn3.164, 2015/03/31. 
Spencer, JI, Bell, JS, DeLuca, GC., 2018. Vascular pathology in multiple sclerosis: reframing pathogenesis around the blood-brain barrier. J. Neurol. Neurosurg. Psychiatry 89, 42-52. https://doi.org/10.1136/jnnp-2017-316011, 2017/09/02.

Valavanidis, A, Vlachogianni, T, Fiotakis, K., 2009. Tobacco smoke: involvement of reactive oxygen species and stable free radicals in mechanisms of oxidative damage, carcinogenesis and synergistic effects with other respirable particles. Int. J. Environ. Res. Public Health 6, 445-462. https://doi.org/10.3390/ijerph6020445, 2009/05/ 15.

Waters, P, Woodhall, M, O'Connor, KC, et al., 2015. MOG cell-based assay detects nonMS patients with inflammatory neurologic disease. Neurol. Neuroimmunol. Neuroinflamm. 2, e89. https://doi.org/10.1212/NXI.0000000000000089, 2015/ 03/31.

Varela F, CF, Rojas, J, et al., 2016. Smoking and Disease Severity in Patients with Neuromyelitis Optica. Neurology.

Waters, PJ, McKeon, A, Leite, MI, et al., 2012. Serologic diagnosis of NMO: a multicenter comparison of aquaporin-4-IgG assays. Neurology 78, 665-671. https://doi.org/ 10.1212/WNL.0b013e318248dec1 discussion 6692012/02/04.
Wingerchuk, DM., 2012. Smoking: effects on multiple sclerosis susceptibility and disease progression. Ther. Adv. Neurol. Disord. 5, 13-22. https://doi.org/10.1177/ 1756285611425694, 2012/01/26.

Yeo, T, Probert, F, Jurynczyk, M, et al., 2019. Classifying the antibody-negative NMO syndromes: Clinical, imaging, and metabolomic modeling. Neurol. Neuroimmunol. Neuroinflamm. 6, e626. https://doi.org/10.1212/NXI.0000000000000626, 2019/ $10 / 30$.

Yin, W, Ngwe, EC, Ghebrehiwet, B, et al., 2015. The combined effect of sidestream smoke and dynamic shear stress on endothelial cell inflammatory responses. Thromb. Res. 135, 362-367. https://doi.org/10.1016/j.thromres.2014.11.018, 2014/12/04.

YANG Y-jX, Ting-ting, AO, Dong-hui, Yang, HE, Yang-yang, WANG, Shan-shan, ZHONG, Jian, WU, Guang-zhi, LIU, 2016. Association of smoking with risk of multiple sclerosis and neuromyelitis optica spectrum disorders. Chinese J. Contemp. Neurol. Neurosurg. 16 (9), 598-602.

Yuan, X, Shan, M, You, R, et al., 2015. Activation of C3a receptor is required in cigarette smoke-mediated emphysema. Mucosal Immunol. 8, 874-885. https://doi.org/ 10.1038/mi.2014.118, 2014/12/04. 